\title{
Desenvolvimento de um Modelo Fuzzy e Algoritmos de Aprendizado para Sequência de Partida de Plantas Industriais
}

\author{
André da Silva Barcelos* Fábio Muniz Mazzoni** \\ * Instituto de Ciência e Tecnologia, Universidade Federal Fluminense, \\ RJ, (e-mail: andrebarcelos813@hotmail.com). \\ ** Instituto de Ciência e Tecnologia, Universidade Federal Fluminense, \\ RJ, (e-mail: fabiomuma@hotmail.com).
}

\begin{abstract}
This paper presents a fuzzy-analytic model to describes the behavior of four natural gas processing plants located in an industrial facilities which can operate in parallel mode, according to the national energy demand. The starting sequence of processing units is a strategic decision problem, that consists of determining which of the units has priority to process natural gas under the bias of economic and operational viability. Thus, this work adopts machine learning algorithms in order to define the sequence of operation in a multivariable model. The implementation of this proposal led to an estimated reduction of waste production in the order of $16 \%$ per month.

Resumo: Neste trabalho é desenvolvido um modelo fuzzy-analítico que descreve o comportamento de quatro unidades de processamento de gás natural, localizadas em um parque industrial e que podem operar em paralelo de acordo com a demanda energética nacional. A sequência de partida de unidades de processamento é um problema de decisão estratégica, que consiste em determinar qual das unidades tem prioridade para processar gás natural sob o viés da viabilidade econômica e operacional. Assim, utiliza-se de algoritmos de aprendizado de máquinas, com intuito de determinar a melhor sequência através de um modelo multivariável. A implementação desta proposta acarretou em uma estimativa de redução de desperdícios na produção na ordem de $16 \%$ ao mês.
\end{abstract}

Keywords: Natural gas; Machine learning; Fuzzy; Intelligent decision making Palavras-chaves: Gás natural; Aprendizagem de máquinas; Fuzzy; Tomada de decisão inteligente.

\section{INTRODUÇÃO}

As unidades de processamento de gás natural (UPGN's) que estão localizadas em parques industriais, são instalações que recebem o gás natural (GN) como insumo e tem a finalidade de produzir gás natural processado (GNP). Desta forma, os parques industriais utilizam-se de uma ou mais UPGN's em produção paralela para atender a demanda nacional de GNP (Perlotti et al., 2016).

Neste cenário, faz-se necessário definir a sequência de partida das UPGN's, onde considera-se as condições de operação de cada unidade (Gholian et al., 2013). Isto posto, o trabalho tem por objetivo desenvolver um modelo do comportamento de quatro UPGN's dentro de um parque industrial.

Este modelo de comportamento será utilizado por algoritmos de classificação, com a finalidade de determinar o sequenciamento de partida das UPGN's de acordo com a demanda de GNP. Para a elaboração do modelo, a seção 2 apresenta um resumo do processamento de gás natural com a finalidade de apontar as variáveis, funções e considerações sobre o comportamento das UPGN's.

A primeira função que consta no modelo é a estabilidade operacional, que depende das condições de automação, controle e equipamentos da planta industrial e será descrita por lógica fuzzy. As funções de vazão, eficiência da produção e desperdício de insumos são obtidas analiticamente no domínio do tempo. Por fim, a função de operacionalidade é modelada por lógica fuzzy, onde considera-se os aspectos de layout, construção e recursos da UPGN.

Na seção 3, são apresentados os algoritmos de classificação que são: O Technique for Order Preference by Similarity to a Ideal Solution (TOPSIS); Rede Neural Artificial (RNA); Máquina de Vetor Suporte (MVS) e Regressão Logistíca (RegLog). Os experimentos encontram-se descritos na seção 4 , a seção 5 analisa os resultados das simulações e a seção 6 apresenta a conclusão.

\footnotetext{
^ Os autores agradecem o apoio da Petrobras.
} 


\section{DESENVOLVIMENTO DO MODELO}

\subsection{Processamento de gás natural}

A indústria de beneficiamento do GN utiliza-se de UPGN's em paralelo que devem entrar em operação ou interromper a produção de forma independente. Cada uma das UPGN's contém uma torre de processamento que recebe como insumo o GN (composto de hidrocarbonetos), onde o metano $\left(C_{1}\right)$ e o etano $\left(C_{2}\right)$ são às frações de menor peso molecular e maior volatilidade que ascendem até o topo da torre de processamento.

O propano $\left(C_{3}\right)$, butano $\left(C_{4}\right)$ e pentanos $\left(C_{5}+\right)$, encontramse em estado líquido e descem ao fundo da torre de processamento (Kidnay et al., 2011). A Figura 1 mostra o arranjo de quatro UPGN's em um parque industrial.

Figura 1. Fluxograma de Processamento

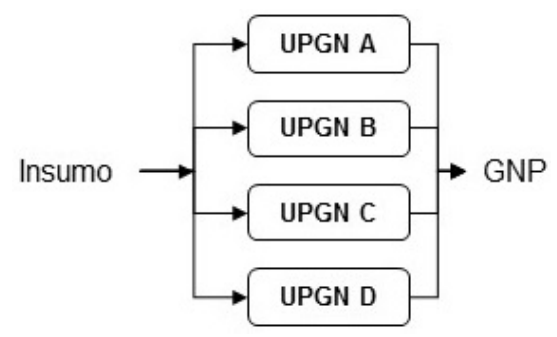

Conforme observa-se na Figura 2, o GNP é composto de $C_{1}$ e $C_{2}$ que ascendem ao topo da torre de processamento e será fornecido ao mercado consumidor, de acordo com a demanda. O líquido de fundo da torre de processsamento é composto de $C_{3}, C_{4}$ e $C_{5}+$ e caracteriza um subproduto que será direcionado para unidades de processamento de líquidos (Mokhatab and Poe, 2012).

Figura 2. Torre de Processamento

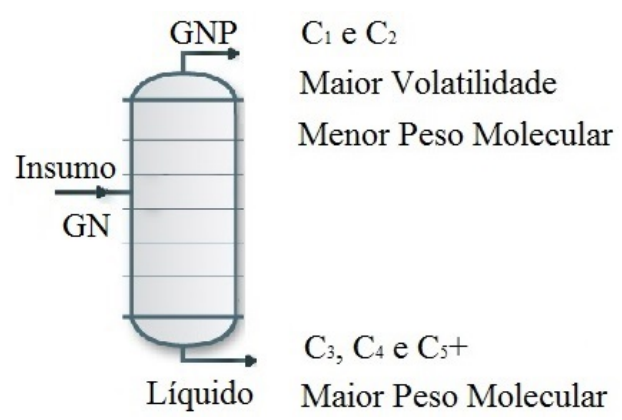

A seguir serão apresentadas as variáveis e funções que determinam o comportamento de uma UPGN para compreender a dinâmica do processo de produção e construir um modelo.

\subsection{Estabilidade Operacional}

Para obter a estabilidade operacional, utiliza-se dos conceitos de grau de automação, capacidade de controle e do percentual de equipamentos em stand-by que define as três variáveis de entrada de uma função que retorna como saída a estabilidade operacional.
O grau de automação é definido como a quantidade de variáveis críticas do processo interno, alarmes e malhas de controle, enquanto a capacidade de controle é determinada pela quantidade de malhas estáveis e controláveis que são capazes de manter os processos termodinâmicos estabilizados. A terceira variável de entrada é a quantidade percentual de equipamentos em standby que remete o potencial de manter a UPGN em operação contínua.

Para obter a estabilidade operacional que é a função de saída, este trabalho utiliza-se da lógica fuzzy e estabelece três superfícies simétricas que avalia as três variáveis de entrada, tomadas duas à duas em relação a estabilidade operacional. A função de pertinência é a trapezoidal com cinco hierarquias (Muito Baixo, Baixo, Adequado, Alto, Muito Alto) e com três superfícies semelhantes à Figura 3.

Figura 3. Superfície da estabilidade operacional

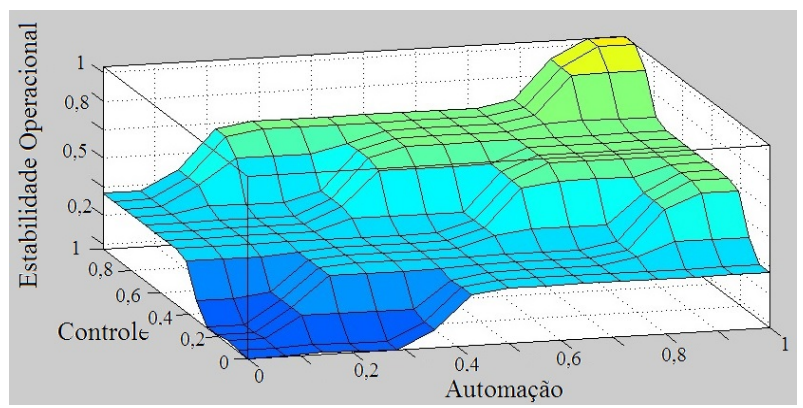

O método de defuzzyficação adotado é o centro de gravidade (COG), que retorna um valor numérico para a estabilidade operacional de acordo com as três variáveis de entrada.

\subsection{Vazão da produção}

Para medir a vazão de produção de GNP $\left(\mu_{p}(t)\right)$, utilizase da integração do volume produzido $v_{p}(t)$ conforme a equação 1 .

$$
\mu_{p}(t)=\int_{t=0}^{t} v_{p}(t) d t
$$

O resultado da equação 1 torna possível obter a vazão máxima $\left(\mu_{p(\max )}\right)$ de produção e por conseguinte construir a Tabela 1. A unidade de processamento A produz a maior vazão de GNP possível $\left(\mu_{p(\max )}=1\right)$ quando comparada com as demais unidades.

Tabela 1. Vazão da produção

\begin{tabular}{l|c|c|c|c}
\hline UPGN & $\mathrm{A}$ & $\mathrm{B}$ & $\mathrm{C}$ & $\mathrm{D}$ \\
$\mu_{\max }$ & 1,0000 & 0,9074 & 0,9074 & 0,9359 \\
\hline
\end{tabular}

\subsection{Eficiência da produção}

A vazão de produção de GNP $\left(\mu_{p}(t)\right)$ e a vazão de insumo $\left(i_{p}(t)\right)$ são utilizadas para medir a eficiência $(\xi(t))$ da produção. Para realizar este cálculo, toma-se a razão da vazão de produção pela integração da vazão de insumo através da equação 2 : 


$$
\xi(t)=\frac{\mu_{p}(t)}{\int_{t=0}^{t} i_{p}(t) d t}
$$

O valor de $\xi(t)$ representa a eficiência em que uma UPGN consegue transformar a fração de $C_{1}$ e $C_{2}$ do GN em produto ao longo do tempo. A Tabela 2 apresenta a eficiência média da produção $\xi_{m e d}$ de cada UPGN.

Tabela 2. Eficiência média da produção

\begin{tabular}{l|c|c|c|c}
\hline UPGN & $\mathrm{A}$ & $\mathrm{B}$ & $\mathrm{C}$ & $\mathrm{D}$ \\
$\xi_{\text {med }}$ & 0,9231 & 0,7058 & 0,7003 & 0,8874 \\
\hline
\end{tabular}

\subsection{Desperdício de insumo}

Define-se como desperdício de insumo $\left(\mu_{d}\right)$ o volume de GN admitido na torre de processamento que por descontrole ou desvios operacionais é impedido de converter-se em produto especificado.

Isto ocorre quando as frações de $C_{3}, C_{4}$ ou $C_{5}+$ ascendem indevidamente ao topo da torre de processamento e entram na composição do GNP. Nesta condição todo o produto que sai pelo topo da torre de processamento encontra-se desespecificado e deve ser reprocessado ou descartado no sistema de rejeito.

O reprocessamento consiste em injetar o produto desespecificado na entrada de insumo da torre e esta manobra acarreta em retrabalho e redução da eficiência da produção. A equação 3 permite obter o volume reprocessado $\left(\mu_{r}\right)$.

$$
\mu_{r}=\int_{t=0}^{t} \mu_{r}(t) d t
$$

O sistema de rejeito é uma instalação industrial com a finalidade de descartar o gás que ascendeu ao topo da torre de processamento e encontra-se desespecificado. Obtém-se por meio da equação 4 a totalização deste volume rejeitado $\left(\mu_{j}\right)$.

$$
\mu_{j}=\int_{t=0}^{t} \mu_{j}(t) d t
$$

O volume de gás desperdiçado é medido por meio da equação 5 e consiste na média harmônica do volume de gás reprocessado com o volume de gás enviado ao sistema de rejeito. A escolha da média harmônica consiste na possibilidade de ponderar o custo do reprocessamento e do rejeito.

$$
\mu_{d}=\frac{2}{1 / \mu_{r}+1 / \mu_{j}}
$$

A Tabela 3 é o resultado da equação 5 e demonstra o volume de desperdício de cada UPGN. Por definição, fazse necessário minimizar esta equação de acordo com as práticas de otimização da produção.
Tabela 3. Volume de desperdício

\begin{tabular}{c|c|c|c|c}
\hline UPGN & $\mathrm{A}$ & $\mathrm{B}$ & $\mathrm{C}$ & $\mathrm{D}$ \\
$\mu_{\text {medio }}$ & 0,1419 & 0,1180 & 0,1312 & 0,1074 \\
\hline
\end{tabular}

\subsection{Operacionalidade}

As UPGN's apresentam diferenças de layout, tecnologia, modelo de construção e particularidades em suas instalações que influenciam a capacidade de partir e operar adequadamente. Para obter a operacionalidade, utiliza-se da lógica fuzzy para criar uma superfície que avalia as três variáveis de entrada que são: o tempo de partida; os recursos necessários e o tempo de estabilização conforme observa-se na Figura 4

Figura 4. Mapa de regras da operacionalidade

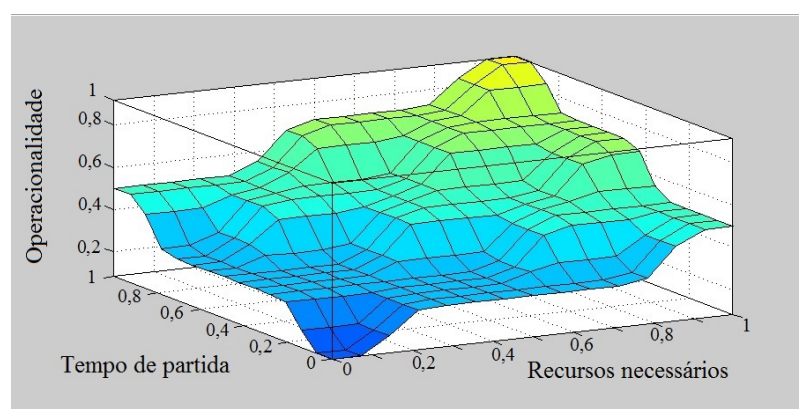

A função de pertinência adotada é a trapezoidal com cinco hierarquias (Muito Baixo, Baixo, Adequado, Alto e Muito Alto) e com três superfícies simétricas relacionadas duas à duas em função da Operacionalidade. O método de defuzzyficação adotado é o centro de gravidade que retorna um valor numérico de acordo com as três variáveis de entrada.

\section{ALGORITMOS PARA CLASSIFICAÇÃO}

Realizou-se testes em algoritmos de aprendizado, regressores, redução dimensional, árvores de decisão e heurísticas com a finalidade de classificar corretamente os resultados do modelo proposto. Por conguinte, entende-se que é possível medir o desempenho de um algoritmo ao realizar uma determinada tarefa e caso seja possível melhorar este desempenho iterativamente, fica determinado que este algoritmo é capaz de aprender pela experiência (Dietterich, 2000).

Os algoritmos que apresentaram os melhores resultados para a classificação do modelo proposto foram o TOPSIS, a rede neural artificial, a máquina de vetor suporte e a regressão logística que estão descritos nas próximas subseções.

\subsection{TOPSIS}

O algoritmo Technique for Order Preference by Similarity to a Ideal Solution (TOPSIS) é definido como um método multicritério, onde mede-se a distância euclidiana entre todas as alternativas possíves com a solução ideal (Şengül et al., 2015). 
Conceitualmente define-se que a melhor alternativa $\left(D^{+}\right)$ encontra-se com a menor distância euclidiana possível para a solução ideal e por conseguinte, a pior alternativa $\left(D^{-}\right)$, encontra-se o mais próximo possível da solucão não ideal (Hwang and Yoon, 1981).

O método TOPSIS avalia a matriz de alternativas da Figura 5 que contém todas as alternativas possíveis $A_{i}$ associadas aos atributos $C_{j}$. (Şengül et al., 2015).

Figura 5. Matriz de alternativas

$$
A=\ldots \begin{array}{cccc}
C_{1} & C_{2} & \ldots & C_{j} \\
A_{1} & A_{i}
\end{array} \quad\left\{\begin{array}{cccc}
x_{11} & x_{12} & \ldots & x_{1 j} \\
\vdots & \ddots & \vdots \\
& & & \\
x_{i 1} & x_{i 2} & \ldots & x_{i j}
\end{array}\right\}
$$

O processo de execução do TOPSIS pode ser expresso em uma sequência de etapas, onde avalia-se a construção da matriz de alternativas ponderadas (Behzadian et al., 2012). A seguir encontram-se as etapas descritas em detalhes.

- Construção da matriz de alternativas ponderadas:

Utiliza-se de um vetor de pesos $\left(w_{1}, w_{2}, \ldots w_{n}\right)$ que deve ser fornecido pelo especialista para ponderar as alternativas em cada critério. Devido à questão cognitiva do ser humano, o especialista pode ponderar as alternativas de maneira incoerente e entende-se que a determinação de pesos torna-se induzida (Zavadskas and Podvezko, 2016).

Uma forma de obter pesos para solucionar problemas onde prevalece a indução humana, é utilizar-se de teorias e métodos que por definição são independentes do especialista. Um desses métodos calcula a medida de incerteza ou entropia do sistema e mede o estado de desordem ou inconsistência em um dado momento (Ormos and Zibriczky, 2014).

O conceito de entropia na atribuição de pesos representa uma medida de estado momentâneo, onde espera-se que quanto maior for a inconsistência do sistema, maior será a entropia (Joshi and Kumar, 2014). Para este trabalho, os pesos $\left(w_{1}, w_{2}, \ldots w_{n}\right)$ são obtidos pela entropia da matriz de alternativas de acordo com as equações 6,7 e 8 .

$$
\begin{gathered}
e_{j}=-\frac{1}{\ln (m)} \sum_{i=1}^{m} x_{i j} \ln \left(x_{i j}\right) \\
d_{j}=1-e_{j} \\
w_{j}=\frac{d_{j}}{\sum_{j=1}^{m} d_{j}}
\end{gathered}
$$

Uma vez que obtenha-se a entropia conforme a equação 6, utiliza-se da equação 7 e 8 para obtenção do vetor de pesos $w_{j}$. A matriz de alternativas ponderadas $v_{i j}$ tem a forma descrita na equação 9 , onde $r_{i j}$ são os valores $\left(x_{i j}\right)$ da matriz de alternativas A normalizada.

$$
v_{i j}=w_{j} r_{i j}
$$

Vale salientar que a matriz ponderada é independente do especialista, pois neste caso realiza-se a determinação dos pesos $\left(w_{i j}\right)$ através da entropia dos valores $\left(x_{i j}\right)$ da matriz A (Yentes et al., 2013).

\section{- Cálculo da similaridade}

Define-se como similaridade a distância euclidiana de cada alternativa à solução ideal. Nesta etapa, calcula-se a similaridade da matriz ponderada, tanto para a solução ideal $\left(D^{+}\right)$, quanto para a solução não ideal $\left(D^{-}\right)$, conforme as equações 10 e 11.

$$
\begin{aligned}
& D_{i}^{+}=\sqrt{\sum_{j=1}^{m}\left[v_{i j}(x)-v_{j}^{+}(x)\right]^{2}} \\
& D_{i}^{-}=\sqrt{\sum_{j=1}^{m}\left[v_{i j}(x)-v_{j}^{-}(x)\right]^{2}}
\end{aligned}
$$

Ao final, utiliza-se da equação 12 para ordenar as alternativas e propor a solução do sequenciamento de partida, onde o vetor $k_{i}$ apresenta um ranking de alternativas obtidas através da entropia da matriz $\mathrm{A}\left(x_{i j}\right)$.

$$
k_{i}=\frac{D_{i}^{-}}{D_{i}^{-}+D_{i}^{+}}
$$

\subsection{Rede Neural Artificial}

A rede neural artificial é uma ferramenta computacional utilizada para classificação de dados com a possibilidade de estabelecer relações não lineares entre entrada e saída (Yegnanarayana, 2009)

Espera-se que as redes neurais tenham a capacidade de aprender através da melhoria de um critério de desempenho por um processo iterativo de ajustes de pesos da rede (Behbahani et al., 2009). A taxa de aprendizagem e os pesos influenciam no tempo de processamento da rede neural e desta forma faz-se necessário definir um critério de parada, onde fica estabelecido que a partir deste momento, a rede encontra-se treinada (Witten et al., 2016).

Durante o treinamento, obtém-se o percentual de acertos na classificação para um tempo de processamento. Ao dobrar-se o tempo de processamento iterativamente, deve ocorrer a convergência do percentual de acertos até um limite temporal definido como critério de parada (Yegnanarayana, 2009).

\subsection{Máquina de Vetor Suporte}

Um algoritmo de máquina de vetor suporte (MVS) disponibiliza os dados no espaço e desenvolve hiperplanos de complexidade ajustável, com a finalidade de separar os dados em categorias distintas (Smola and Schölkopf, 2004). Desta forma, uma MVS pode ser utilizada como um algoritmo multivariável classificador, regressor ou identificador de outliers (Cristianini and Shawe-Taylor, 2000). 
Os hiperplanos são criados de acordo com a base de dados e o algoritmo utiliza-se de uma margem de separação, para permitir uma correta classificação das instâncias ou categorias (Smola and Schölkopf, 2004). Devido ao número de instâncias deste trabalho, faz-se necessário a redução do custo computacional com a finalidade de convergir à solução. Assim, utiliza-se da classificação binária iterativa que propaga erros, porém retorna resultados viáveis (Witten et al., 2016).

\subsection{Regressão Logística}

Um algoritmo que utiliza-se de regressão para classificação encontra aplicação em domínios numéricos, pois entendese que quaisquer técnicas de regressão, pode ser utilizada para classificação (Hosmer Jr et al., 2013). A forma multivariável do algoritmo de RegLog pode ser aplicada em domínios que existem três ou mais possibilidades de classificação, onde os resultados devem ser ordenados (Menard, 2002).

Outrossim, admite-se que os valores de máximo e mínimo de uma base de dados situam-se no limite superior e inferior das escalas. Por conseguinte, atualizam-se os parâmetros da curva iterativamente, para que a função logística torne-se convergente e ajustada aos dados durante a fase de treinamento (Witten et al., 2016).

\section{EXPERIMENTOS}

O modelo descrito nas seções anteriores é representado numericamente em uma matriz $4 \times 5$, onde cada coluna são respectivamente os valores da estabilidade operacional, vazão de produção, eficiência, desperdício de insumo e operacionalidade de cada UPGN. Esta matriz é a representação numérica do modelo do comportamento que varia ao longo do tempo.

Este trabalho utiliza-se do histórico de 1072 matrizes (instâncias) de acordo com o modelo, que representam a partida e operação de plantas reais com intuito de realizar as simulações. Assim, quando um algoritmo computa estas instâncias, espera-se que o resultado seja o sequenciamento de partida.

O sequenciamento indicado pelo algoritmo é representado através de uma palavra, na qual a sequência de letras está disposta em ordem de preferência, como por exemplo a palavra cbda onde a UPGN C tem a prioridade da partida seguida da B e assim, sucessivamente. Isto posto, fica estabelecido que o modelo contém 20 entradas e 24 saídas e que o algoritmo utiliza-se de 1072 instâncias obtidas através de histórico.

Cada algoritmo contém parâmetros que podem ser ajustados iterativamente para melhorar o desempenho da classificação em detrimento do aumento do tempo de computação. Estes ajustes de parâmetros são realizados em simulações sucessivas através da redução do erro relativo.

Uma vez que não ocorra redução de erro relativo em duas simulações sucessivas ou melhoria do desempenho com ajuste de parâmetros, fica determinado que atingiu-se o critério de parada da simulação.

\subsection{TOPSIS}

O algoritmo TOPSIS retorna 771 instâncias classificadas corretamente e observa-se resultados ambíguos. Este fato reside na forma em que o algoritmo classifica suas instâncias e busca soluções.

Uma vez que o método baseia-se em ordenar soluções que se encontram na menor distância da solução ideal e por conseguinte na maior distância da solução não ideal. Fica evidente que em uma base de dados podem existir instâncias com os módulos aproximadamente iguais para a solução ideal e não ideal, pórem com sentidos distintos.

A ordenação ambígua do método TOPSIS é imperceptível em modelos de ordem três ou com número de instâncias reduzidos (10 a 20). Porém quando a ordem do modelo aumenta e o número de instâncias ultrapassa algumas centenas o algoritmo apresenta ambiguidade.

\subsection{Regressão Logística (RegLog)}

Este algoritmo retorna 994 (92,72\%) instâncias classificadas corretamente e aponta as palavras bcad e bacd como as que apresentam os maiores erros de classificação, com nove e sete instâncias respectivamente.

Ao observar a computação deste algoritmo, percebe-se que por seis vezes as instâncias que deveriam ser classificadas como bcad, são classificadas como bacd. Por outro lado, as instâncias que deveriam ser classificadas como bacd, são classificadas como bcad quatro vezes.

Fica evidente que quando a palavra inicia-se por b e termina com d, as letras do meio apresentam ambiguidade na classificação. Como experimento removeu-se as palavras bacd e bcad da classificação e o resultado da simulação atingiu o $94,87 \%$ de acertos.

\subsection{Rede Neural Artificial (RNA)}

Este algoritmo consegue classificar 1001 (93,37\%) instâncias corretamente após 65 simulações, onde realizam-se todos os ajustes necessários aos parâmetros da rede.

A avaliação dos resultados mostra que a instância que deveria ser classificada como dcba é classificada incorretamente em cinco ocasiões como cdba. Por outro lado, a palavra cbda é classificada como dcba seis vezes e fica evidente que a rede apresenta uma região de ambiguidade para estas duas palavras.

\subsection{Máquina de Vetor Suporte (MVS)}

Este algoritmo classifica 1034 (96,45\%) instâncias corretamente após 225 simulações com tempo de processamento na ordem de horas. A região ambígua para este algoritmo situa-se nas palavras cbda e bcda, com quatro e três classificações erradas respectivamente.

\section{ANÁLISE DOS RESULTADOS}

Este trabalho utiliza-se de quatro métricas para a análise dos resultados e validação da simulação. A primeira métrica é o percentual de instâncias classificadas corretamente por cada algoritmo (\%cts) enquanto que a segunda 
métrica é a raiz quadrada do erro médio da melhor classificação (rqm).

A terceira métrica é o número total de simulações completas que permitiram o ajuste iterativo de pesos e consequentemente, o aumento de desempenho da rede (nts). Por fim, a quarta métrica é o tempo total de processamento de todas as simulações (tts). Na Tabela 4 encontra-se um resumo das métricas adotadas.

Tabela 4. Métrica adotadas

\begin{tabular}{l|c|c|c|c}
\hline Métrica & \%cts & rqm & nts & tts \\
\hline TOPSIS & 71,92 & 0.0961 & 60 & $00: 15: 25$ \\
RegLog & 92,72 & 0.1380 & 163 & $00: 56: 25$ \\
RNA & 93,37 & 0.0816 & 65 & $31: 12: 15$ \\
MVS & 96,45 & 0.1925 & 225 & $01: 15: 20$ \\
\hline
\end{tabular}

Observa-se que a MVS apresenta o maior percentual de acertos para a classificação correta das instâncias, em um tempo total de simulação em aproximadamente 1 hora e 15 minutos. Por outro lado, a rede neural artificial necessita de um tempo de simulação próximo de 32 horas para conseguir um resultado inferior à MVS.

A raiz quadrada do erro médio (rqm) leva em conta todas as classificações das instâncias erradas e pondera sobre o quanto estariam distantes das instâncias corretas. Assim, afirma-se que os erros de classificação cometidos pelo algoritmo MVS tem maior magnitude que os erros cometidos pelos demais algoritmos.

\section{CONCLUSÃO}

A decisão do sequenciamento de partida de plantas industriais é estratégica para as empresas inseridas na atividade de processamento, pois trata-se da produção de gás natural em um cenário de demanda nacional. Esta responsabilidade fica à cargo dos gestores destas unidades que devem determinar o sequenciamento de partida empiricamente.

Desta forma, este trabalho propõe um modelo fuzzyanalítico, que utiliza-se de fatores operacionais e econômicos para determinar um modelo comportamento das UPGN's e definir o sequenciamento de partida, utilizandose do algoritmos de máquina de vetor suporte.

Outra análise que pode ser realizada é que com este modelo, torna-se possível prever as deficiências operacionais de cada unidade e gerenciar recursos para investimentos ou manutenção com a finalidade de melhorar o desempenho na produção e operação. Este trabalho encontra-se em andamento onde estima-se (baseado em histórico) que o lucro cessante encontra-se minimizado com uma redução do desperdício na ordem de $16 \%$ ao mês.

Como sugestão para trabalhos futuros, existe a possibilidade de aplicação para diferentes tipos de plantas industriais. Um exemplo caraterístico são os parques de compressão de gás natural e bombeamento de petróleo, que contém bombas industriais de grande porte e turbinas à gás que operam em paralelo e são responsáveis pelo envio de produto ao mercado consumidor.

\section{REFERÊNCIAS}

Behbahani, R.M., Jazayeri-Rad, H., and Hajmirzaee, S. (2009). Fault detection and diagnosis in a sour gas absorption column using neural networks. Chemical engineering \& technology, 32(5), 840-845.

Behzadian, M., Otaghsara, S.K., Yazdani, M., and Ignatius, J. (2012). A state-of the-art survey of topsis applications. Expert Systems with applications, 39(17), 13051-13069.

Cristianini, N. and Shawe-Taylor, J. (2000). An introduction to support vector machines and other kernel-based learning methods. Cambridge university press.

Dietterich, T.G. (2000). Ensemble methods in machine learning. In International workshop on multiple classifier systems, 1-15. Springer.

Gholian, A., Mohsenian-Rad, H., Hua, Y., and Qin, J. (2013). Optimal industrial load control in smart grid: A case study for oil refineries. In 2013 IEEE Power \&6 Energy Society General Meeting, 1-5. IEEE.

Hosmer Jr, D.W., Lemeshow, S., and Sturdivant, R.X. (2013). Applied logistic regression, volume 398. John Wiley \& Sons.

Hwang, C.L. and Yoon, K. (1981). Methods for multiple attribute decision making. In Multiple attribute decision making, 58-191. Springer.

Joshi, D. and Kumar, S. (2014). Intuitionistic fuzzy entropy and distance measure based topsis method for multi-criteria decision making. Egyptian informatics journal, 15(2), 97-104.

Kidnay, A.J., Parrish, W.R., and McCartney, D.G. (2011). Fundamentals of natural gas processing. CRC press.

Menard, S. (2002). Applied logistic regression analysis, volume 106. Sage.

Mokhatab, S. and Poe, W.A. (2012). Handbook of natural gas transmission and processing. Gulf professional publishing.

Ormos, M. and Zibriczky, D. (2014). Entropy-based financial asset pricing. PloS one, 9(12), e115742.

Perlotti, E.A., Santos, E.M.d., and Costa, H.C.d.M. (2016). Concentração espacial da indústria de são paulo: evidências sobre o papel da disponibilidade de gás natural. Estudos avançados, 30(87), 143-164.

Şengül, Ü., Eren, M., Shiraz, S.E., Gezder, V., and Şengül, A.B. (2015). Fuzzy topsis method for ranking renewable energy supply systems in turkey. Renewable Energy, 75, 617-625.

Smola, A.J. and Schölkopf, B. (2004). A tutorial on support vector regression. Statistics and computing, 14(3), 199-222.

Witten, I.H., Frank, E., Hall, M.A., and Pal, C.J. (2016). Data Mining: Practical machine learning tools and techniques. Morgan Kaufmann.

Yegnanarayana, B. (2009). Artificial neural networks. PHI Learning Pvt. Ltd.

Yentes, J.M., Hunt, N., Schmid, K.K., Kaipust, J.P., McGrath, D., and Stergiou, N. (2013). The appropriate use of approximate entropy and sample entropy with short data sets. Annals of biomedical engineering, 41(2), 349-365.

Zavadskas, E.K. and Podvezko, V. (2016). Integrated determination of objective criteria weights in mcdm. International Journal of Information Technology $\&$ Decision Making, 15(02), 267-283. 